\title{
Protective Effect of Alpha Lipoic Acid against Phenytoin Induced Behavioral Abnormalities in Rats
}

\section{Saraswathy $\mathbf{G R}^{1 *}$, Maheswari $\mathrm{E}^{2}$ and Santhrani $\mathrm{T}^{3}$}

${ }^{1}$ Department of Pharmacology, M.S. Ramaiah University of Applied Sciences, Karnataka, India

${ }^{2}$ Department of Pharmacy Practice, M.S. Ramaiah University of Applied Sciences, Karnataka, India

${ }^{3}$ Department of Pharmacology, Institute of Pharmaceutical Technology, Sri Padmavathi Mahila Visvavidyalayam, Andhra Pradesh, India

\begin{abstract}
Background: Long term administration of antiepileptic drug phenytoin is reported to cause behavioral abnormalities mediated via oxidative stress. The effect of an antioxidant alpha lipoic acid (ALA) against phenytoin induced behavioral abnormalities was investigated.

Methods: The study was carried out in albino wistar rats. The rats were divided into five groups of six animals each. Group 1 received $0.2 \%$ carboxy methyl cellulose (CMC, p.o), group 2 received $20 \mathrm{mg} / \mathrm{kg}$ phenytoin (p.o), groups 3,4 and 5 received 50,100 and $200 \mathrm{mg} / \mathrm{kg}$ (p.o) of ALA in $0.2 \%$ CMC, respectively $1 \mathrm{~h}$ prior to phenytoin for 45 days. Motor coordination, exploratory behavior, memory and spontaneous motor activity were evaluated by Rota rod, Hole board, Elevated plus maze and Actophotometer respectively. On day 45, regional brain lipid peroxidation and acetylcholinesterase (ACh E) activity along with brain histopathological investigation were performed after euthanasia. In addition, pharmacokinetic and pharmacodynamic drug interactions between phenytoin and ALA were also studied. Results: Long term administration of phenytoin showed behavioral abnormalities, increased regional brain malondialdehyde (MDA) and ACh E activity. The histopathological investigation showed congested and damaged cells in brain regions. ALA substantially reversed phenytoin induced behavioral abnormalities, oxidative stress and alleviated the histopathological abnormalities. There were no significant differences in the serum levels of phenytoin and the degree of protection offered by phenytoin in ALA supplemented groups revealing that there were no pharmacokinetic and pharmacodynamic interactions between phenytoin and ALA.
\end{abstract}

Conclusion: This study reports the effectiveness of ALA against phenytoin induced behavioral abnormalities and oxidative stress in rats without altering the bioavailability of phenytoin and its therapeutic effect.

Keywords: Phenytoin; Alpha lipoic acid; Oxidative stress; Behavioral abnormalities

\section{Introduction}

Epileptic disorders affect approximately $0.5-1 \%$ of human population [1]. The main goal in the treatment of epilepsy is to attain a complete control over seizures without potential side effects to improve the quality of life. Phenytoin is a most common and effective antiepileptic drug (AED) prescribed for a prolonged period to achieve seizure control in all types of generalized as well as partial seizures and status epilepticus [2]. Phenytoin causes several serious side effects notably neurotoxicity [3]. This drug generates reactive oxygen species during its metabolism leading to severe oxidative stress which in turn results in neuro-degeneration. Long term phenytoin therapy causes cerebellar degeneration [4] resulting in cognitive impairment [5], ataxia, nystagmus and slurred speech [6].

Alpha-lipoic acid (ALA) also known as thioctic acid (TA), is essential for the function of different enzymes of oxidative metabolism $[7,8]$. ALA was initially used in the treatment of acute poisoning with amanita phalloides, deadly poison followed by its application in treating neuropathic complaints [9]. It is believed that ALA or its reduced form, dihydrolipoic acid (DHLA) possess a number of biochemical functions acting as biological antioxidants, as metal chelators, reducing the oxidized forms of other antioxidant agents such as vitamin $\mathrm{C}$ and $\mathrm{E}$ and glutathione (GSH). ALA has also shown to improve endothelial dysfunction [10] and to reduce oxidative stress post exercise training [11]. It also protects against the development of atherosclerosis and inhibits the progression of an already established atherosclerosis plaque $[12,13]$. These above-mentioned benefits have insisted the use of ALA as a potential therapeutic agent for many chronic diseases with great epidemiological as well as economic and social impact such as diabetes mellitus (DM) and its complications [14,15], hypertension [16], Alzheimer's disease (AD) [17], Down syndrome [18], cognitive dysfunction and some types of cancer [19]. ALA is recommended as a dietary supplement in medical and nutritional management of patients.

The therapeutic or toxic effects of phenytoin depend on its serum concentration. The serum levels of phenytoin were estimated at the end of the study period after the steady state of the drug was achieved to investigate if there were any pharmacokinetic interactions between phenytoin and ALA. Pharmacodynamic interference of ALA over antiepileptic protection offered by phenytoin was also studied.

Our work is a preliminary study to assess the ameliorative effect of ALA against phenytoin induced behavioral abnormalities. Phenytoin and its metabolites are reported to induce oxidative stress in brain regions leading to behavioral abnormalities. Hence, we explored the ameliorative effect of ALA against phenytoin induced behavioral abnormalities like impaired cognition, exploratory behavior, spontaneous motor activity and locomotor activity in addition to the estimation of regional brain lipid peroxidation and acetyl cholinesterase

${ }^{*}$ Corresponding author: Dr. Saraswathy G.R., Department of Pharmacology, M.S. Ramaiah University of Applied Sciences, Bangalore, Karnataka, India, Tel: 08123230400; Fax: 080-23607488; E-mail: saraswathypradish@gmail.com

Received July 08, 2015; Accepted July 28, 2015; Published July 31, 2015

Citation: Saraswathy GR, Maheswari E, Santhrani T (2015) Protective Effect of Alpha Lipoic Acid against Phenytoin Induced Behavioral Abnormalities in Rats. J Mol Biomark Diagn 5: 241. doi:10.4172/2155-9929.1000241

Copyright: (c) 2015 Saraswathy GR, et al. This is an open-access article distributed under the terms of the Creative Commons Attribution License, which permits unrestricted use, distribution, and reproduction in any medium, provided the original author and source are credited 
(ACh E) activity. Antioxidant supplementation is expected to offer a better antiepileptic therapy with minimal or no toxicity, which may improve the quality of life in patients under phenytoin treatment.

\section{Materials and Methods}

\section{Animals}

Adult male albino rats weighing 150-200 g were maintained at room temperature $\left(25 \pm 3^{\circ} \mathrm{C}\right)$, fed with a rodent lab diet and tap water ad libitum. The study protocol was approved by the Institutional Animal Ethical Committee of M.S. Ramaiah College of Pharmacy, Bangalore, Karnataka, India, Ref. No.220/abc/CPCSEA.

\section{Study protocol}

The rats were divided into five groups consisting of six animals each. First group served as control and received $0.2 \%$ carboxy methyl cellulose (CMC) (p.o) for 45 days. Second group received $20 \mathrm{mg} / \mathrm{Kg}$ phenytoin dissolved in $0.2 \% \mathrm{CMC}$ (p.o) for 45 days. Third, fourth and fifth group received 50, 100 and $200 \mathrm{mg} / \mathrm{kg}$ of ALA in $0.2 \%$ CMC (p.o) respectively $1 \mathrm{~h}$ prior to administration of $20 \mathrm{mg} / \mathrm{Kg}$ phenytoin for 45 days.

\section{Evaluation of behavioral abnormalities}

The behavioral parameters were analyzed $2 \mathrm{hrs}$ after the administration of ALA and phenytoin. Memory, motor co-ordination, locomotor activity and exploratory behavior were assessed on $0,15^{\text {th }}$, $30^{\text {th }}$ and $45^{\text {th }}$ day. On $45^{\text {th }}$ day, behavioral tests were carried out, $3 \mathrm{~h}$ after phenytoin administration (steady state concentration) and phenytoin with ALA supplementation the animals were subjected to maximal electro shock (MES) induced convulsions to compare the degree of protection offered by phenytoin in phenytoin treated group and groups subjected to combination of phenytoin and antioxidants. Immediately after MES the animals were decapitated under ether anesthesia, blood was collected from retro-orbital plexus to estimate the serum levels of phenytoin. The brains were quickly removed and differentiated into cortex, mid brain, medulla, pons and cerebellum and were subjected to assessment of the extent of lipid peroxidation and acetylcholinesterase (ACh E) activity.

\section{Motor co-ordination test}

Motor co-ordination test was conducted in rats using a RotaRod apparatus (Inco-Ambala, India). The animals were screened for motor co-ordination and the animals which stayed on the rotating rod without falling for $120 \mathrm{sec}$ were chosen for the study. Each animal was placed on the Rota rod and the time taken by the animal to fall down was noted [20].

\section{Test for locomotor activity}

Spontaneous motor activity was monitored using Actophotometer. Each animal was subjected to an adaptation period of 2-5 minutes after which their locomotor activity was assessed for 5 minutes. Increase in count was regarded as CNS stimulant activity. Decrease in count was considered as CNS depressant activity [20].

\section{Test for memory impairment}

Elevated plus maze test was used for the assessment of memory. The elevated plus maze consists of two closed arms and two open arms forming a cross, with a quadrangular center and has a height of $50 \mathrm{~cm}$. The rats were placed individually at the end of one open arm facing away from central platform and the time it took to move from the open arm to either of the enclosed arms (transfer latency) was recorded on the day of acquisition trial. Transfer latency is the time taken by the rats to move from one end of the open arm to enclosed arm. The rat was allowed to move freely in the plus maze regardless of open and closed arms for $10 \mathrm{sec}$ after the measurement of transfer latency. The rat was then gently taken out of the plus maze and was returned to its home cage. On the test day, the transfer latency test was performed in the same manner as in the acquisition trial [21].

\section{Test for alertness (Exploratory Behavior)}

$0.5 \mathrm{~m}^{3}$ wooden board with 16 holes $(3 \mathrm{~cm}$ in diameter) was employed for the study. Each rat was placed individually on the board for a period of 6 minutes. In first 2 minutes the animal was allowed for acclimatization and then the number of head dipping performed in the next 4 minutes was noted for each animal [22].

\section{Assessment of oxidative stress in brain tissues}

The brain samples were quickly removed, cleaned with chilled saline, dissected into cortex, midbrain, medulla, pons and cerebellum [23], were stored at $-40^{\circ} \mathrm{C}$.

\section{Estimation of lipid peroxidation in brain regions}

The extent of lipid peroxidation in tissues was assessed by measuring the level of malondialdehyde (MDA) according to the method of Ohkawa, et al. Briefly, $1 \mathrm{ml}(10 \%)$ tissue homogenate was added to the reaction mixture containing $1 \mathrm{ml}$ of trichloro acetic acid (15\%) and $2 \mathrm{ml}$ of thiobarbituric acid $(0.38 \%)$. The reaction mixture was heated for $60 \mathrm{~min}$ at $90^{\circ} \mathrm{C}$, cooled and centrifuged at $6900 \mathrm{rpm}$ for $15 \mathrm{~min}$. The absorbance of supernatant was measured at $532 \mathrm{~nm}$ against blank, which contained all reagents except homogenate. MDA was quantified and expressed as $\mu \mathrm{mol}$ of MDA per mg of wet tissue [24].

\section{Estimation of acetylcholine esterase activity in brain regions}

Acetylthiocholine iodide was used as a synthetic substrate for the assay of ACh E, replacing the natural substrate acetylcholine (ACh). This enzyme hydrolyses the substrate to yield acetate and thiocholine. The free thiol group of thiocholine reacts with 5,5'-dithio-bisnitrobenzoic acid (DTNB) (Ellman's reagent) included in the assay mixture, producing the yellow 4-nitrothiolate anion. The release of this yellow anion is measured at $412 \mathrm{~nm}$. The reaction mixture $(2 \mathrm{~mL}$ final volume) contained $100 \mathrm{mM}$ potassium phosphate buffer, $\mathrm{pH} 7.5$ and 1 $\mathrm{mM}$ DTNB. The method is based on the formation of the yellow anion, 5,5'-dithio-bis-acid nitrobenzoic, measured by absorbance at $412 \mathrm{~nm}$ during 2 -min incubation at $25^{\otimes} \mathrm{C}$. The enzyme was pre-incubated for $2 \mathrm{~min}$. The reaction was initiated by adding $0.8 \mathrm{mM}$ acetylthiocholine iodide [25].

\section{Histopathological investigation on brain tissues}

Brain tissues were dissected out carefully and were kept in 10\% formalin solution prepared with normal saline. Tissues were stained using Hematoxylin and Eosin stain [26].

\section{Maximal electroshock induced seizures (MES)}

Electroconvulsions were induced by ear electrodes (current intensity- $150 \mathrm{~mA}$, duration - $0.2 \mathrm{sec}$ ). The animals were observed for tonic hind limb extension i.e., the hind limbs of animals outstretched $180^{\circ}$ to the plane of the body axis [21].

\section{Estimation of plasma phenytoin concentration by HPLC method}

Chromatographic conditions: Mobile phase consisting of methanol: water: glacial acetic acid (67:33: $1 \mathrm{v} / \mathrm{v} / \mathrm{v})$ was prepared and 
mixed thoroughly, degassed and was used for the HPLC analysis. 1.0 $\mathrm{ml}$ per minute flow rate was maintained throughout the analysis. The eluent was monitored using a UV-VIS detector set at $230 \mathrm{~nm}$ and sensitivity was set at 0.001 a.u.f.s.

\section{Preparation of standard graph}

Standard solutions: Stock solution of $100 \mu \mathrm{g} / \mathrm{ml}$ of phenytoin was prepared in methanol and diluted with methanol to the required concentration. The solutions were stored at $-4^{\circ} \mathrm{C}$. For standard graph 2 , $4,6,8,10,12,14,16,1820 \mu \mathrm{g} / \mathrm{ml}$ of pure phenytoin was used

\section{Plasma extraction}

To each $100 \mu \mathrm{l}$ of plasma sample, $25 \mu \mathrm{l}$ of internal standard (100 $\mu \mathrm{g} / \mathrm{ml}$ carbamazepine solution) was added and extracted with $1.7 \mathrm{ml}$ of ethyl acetate, vortexed for $1 \mathrm{~min}$ and centrifuged at 13,000 rpm for $8 \mathrm{~min}$. The supernatant was evaporated to dryness, the residue was reconstituted with $100 \mu \mathrm{l}$ of mobile phase, vortexed for $1 \mathrm{~min}$. and $20 \mu \mathrm{l}$ was injected onto $\mathrm{C} 18$ column. The retention times were $4.49 \mathrm{~min}$. and $5.15 \mathrm{~min}$. for phenytoin and carbamazepine respectively. The peak area obtained at different concentrations of the drug was plotted against the concentrations of the drug [27].

\section{Statistical analysis}

The results were expressed as mean \pm SEM of each group. One way analysis of variance (ANOVA) followed by the Tukey's post hoc test was used to assess the differences among treatment groups. Statistical analysis was performed using GraphPad Instat software. $\mathrm{p}<0.05$ was considered significant.

\section{Results}

There were no significant differences in transfer latency, exploratory activity, motor coordination and spontaneous motor activity between control, phenytoin alone and phenytoin with ALA pretreated groups on 0 day of the study.

\section{Effect of Alpha Lipoic Acid on phenytoin induced memory impairment}

The effect of chronic treatment of phenytoin and phenytoin along with ALA on memory is shown in Figure 1. There was no significant difference in the transfer latency of the control, phenytoin and phenytoin with ALA $(50,100,200 \mathrm{mg} / \mathrm{Kg}$ ) pretreated groups on the 0 day of the study. The retention transfer latencies increased from $34 \pm$ $0.36 \mathrm{sec}\left(0\right.$ day) to $123.6 \pm 1.22 \mathrm{sec}\left(45^{\text {th }}\right.$ day) $(\mathrm{p}<0.001)$ in phenytoin treated animals. Co-administration of ALA in all the three doses significantly reduced the transfer latency from $15^{\text {th }}$ day till $45^{\text {th }}$ day. The values decreased from $123.6 \pm 1.22 \mathrm{sec}$ in the phenytoin treated group to $93.66 \pm 0.55 \mathrm{sec}(\mathrm{p}<0.001), 80.66 \pm 1.49 \mathrm{sec}(\mathrm{p}<0.001)$ and $73.66 \pm$ $0.76 \mathrm{sec}(\mathrm{p}<0.001)$ in ALA 50, 100 and $200 \mathrm{mg} / \mathrm{Kg}$ co-administered groups respectively on $45^{\text {th }}$ day of the study. ALA at all the three doses produced significant reversal of phenytoin induced memory impairment in a dose dependent fashion but the values did not reach the normal.

\section{Effect of Alpha Lipoic Acid on phenytoin impaired exploratory activity}

There was no significant difference in the exploratory activity of the control, phenytoin treated and phenytoin with ALA (50, 100, $200 \mathrm{mg} /$ $\mathrm{Kg}$ ) pre-treated groups on the 0 day of study. The exploratory activity was assessed by the number of head dippings into the holes of the hole board apparatus. The number of the head dippings decreased from 21 \pm 0.32 ( 0 day) to $3.16 \pm 0.47$ ( $45^{\text {th }}$ day) $(\mathrm{p}<0.001)$ in phenytoin treated animals. Co-administration of ALA in all the three doses significantly increased the exploratory movements from $15^{\text {th }}$ day till $45^{\text {th }}$ day. The number of head dippings increased from $3.16 \pm 0.47$ in the phenytoin

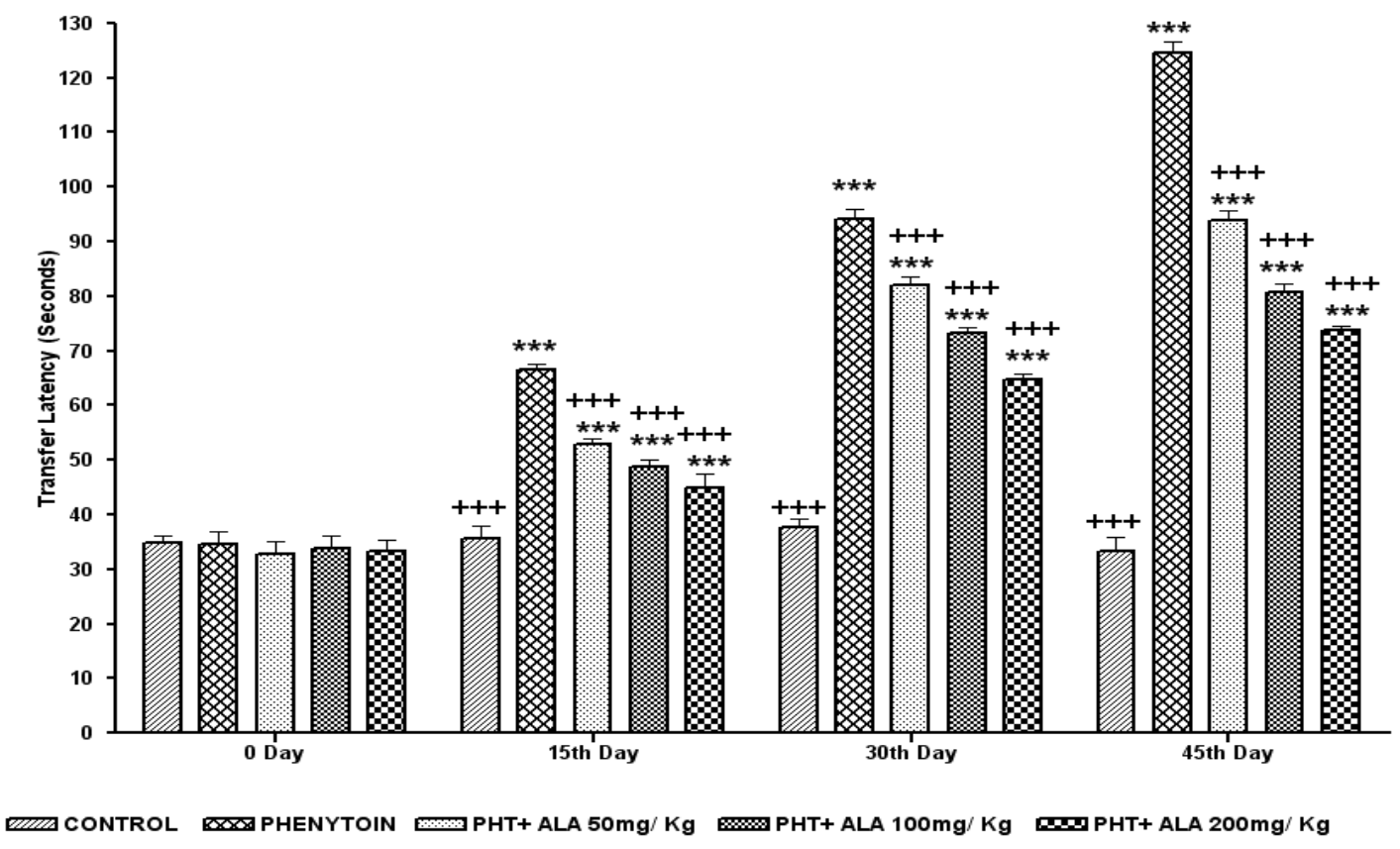

Values are expressed as mean \pm SEM of 6 animals.

${ }^{* * *}(p<0.001),{ }^{* *}(p<0.01),{ }^{*}(p<0.05)$ Vs Control group $+++(p<0.001),++(p<0.01),+(p<0.05)$ Vs Phenytoin group

Figure 1: Effect of ALA on phenytoin induced memory impairment. 
treated group to $8.83 \pm 0.3(\mathrm{p}<0.001), 12.16 \pm 0.47(\mathrm{p}<0.001)$ and 13.5 $\pm 0.42(\mathrm{p}<0.001)$ in ALA 50, 100 and $200 \mathrm{mg} / \mathrm{Kg}$ co-administered groups respectively on $45^{\text {th }}$ day of the study. ALA at all the three doses produced significant reversal of phenytoin impaired exploratory behaviour in a dose dependent manner but the values did not reach the normal values (Figure 2).

\section{Effect of Alpha Lipoic Acid on phenytoin induced motor in co-ordination}

There was no significant difference in motor coordination of the control, phenytoin treated and phenytoin with ALA (50, 100, 200 $\mathrm{mg} / \mathrm{Kg}$ ) pre-treated groups on the 0 day of the study. Phenytoin (20 $\mathrm{mg} / \mathrm{Kg}$, p.o.) significantly impaired the Rota Rod performance of rats from the $120 \mathrm{sec}\left(0\right.$ day) to $17.83 \pm 0.94 \mathrm{sec}$ on $45^{\text {th }}$ day ( $\left.\mathrm{p}<0.001\right)$. Coadministration of ALA in all the three doses significantly improved the motor coordination from $15^{\text {th }}$ day till $45^{\text {th }}$ day. The values increased from $17.83 \pm 0.94 \mathrm{sec}$ in the phenytoin treated group to $54.16 \pm 1.24 \mathrm{sec}$ $(\mathrm{p}<0.001), 87.5 \pm 1.11 \mathrm{sec}(\mathrm{p}<0.001)$ and $93.163 \pm 0.9 \mathrm{sec}(\mathrm{p}<0.001)$ in ALA 50, 100 and $200 \mathrm{mg} / \mathrm{Kg}$ co-administered groups respectively on $45^{\text {th }}$ day of the study. ALA at all the three doses produced significant reversal of phenytoin impaired muscle grip in a dose dependent fashion but the values did not reach the normal (Figure 3).

\section{Effect of Alpha Lipoic Acid on phenytoin impaired locomotor activity}

There was no significant difference in spontaneous motor activity of the control, phenytoin and phenytoin with ALA (50, 100, $200 \mathrm{mg} /$ $\mathrm{Kg}$ ) pre-treated groups on zero day of study. Phenytoin $20 \mathrm{mg} / \mathrm{Kg}$, p.o., significantly decreased the spontaneous motor activity by reducing the performance of the rats on Actophotometer. The count reduced from $306.33 \pm 2.4$ ( 0 day) to $86.16 \pm 1.49$ ( $45^{\text {th }}$ day) $(\mathrm{p}<0.001)$. Coadministration of ALA in all the three doses significantly improved the spontaneous activity from $15^{\text {th }}$ day till $45^{\text {th }}$ day. The values increased from $86.16 \pm 1.49$ in the phenytoin treated group to $112 \pm 1.94$ ( $\mathrm{p}<$ $0.001), 160.8 \pm 3.0(\mathrm{p}<0.001)$ and $209.165 \pm 2.27(\mathrm{p}<0.001)$ in Vit C 50, 100 and $200 \mathrm{mg} / \mathrm{Kg}$ co-administered groups respectively on $45^{\text {th }}$ day of the study. ALA at all the three doses produced significant reversal of phenytoin impaired locomotor activity in a dose dependent fashion but the values did not reach the normal values (Figure 4).

Effect of Alpha lipoic Acid on phenytoin induced alterations in regional brain lipid peroxidation

Phenytoin significantly elevated the lipid peroxidation in medulla, pons, midbrain, cerebellum and cortex. ALA significantly reduced ( $p<0.001)$ the phenytoin induced lipid peroxidation in medulla, pons, midbrain, cerebellum and cortex dose dependently but the values did not reach the normal values when compared with the control group (Figure 5).

\section{Effect of Alpha Lipoic Acid on phenytoin induced alterations} in regional brain AchE activity

Phenytoin exhibited a significant increase in AchE activity in medulla, pons, midbrain, cerebellum and cortex. ALA significantly reduced $(\mathrm{p}<0.001)$ the activity of AchE in medulla, pons, midbrain, cerebellum and cortex dose dependently and brought back the values near to the normal when compared with the control group (Figure 6).

\section{Effect of phenytoin on regional brain histopathology}

Figure 7 Illustrates the effect of phenytoin on brain. Control group showed normal brain architecture (Figure 7a). Phenytoin treated group revealed severe necrosis in cortex (Figure $7 \mathrm{~b}$ ).

\section{Effect of Alpha Lipoic Acid on phenytoin induced alterations} in regional brain histopathology

Figure 8 shows the influence of ALA on phenytoin induced

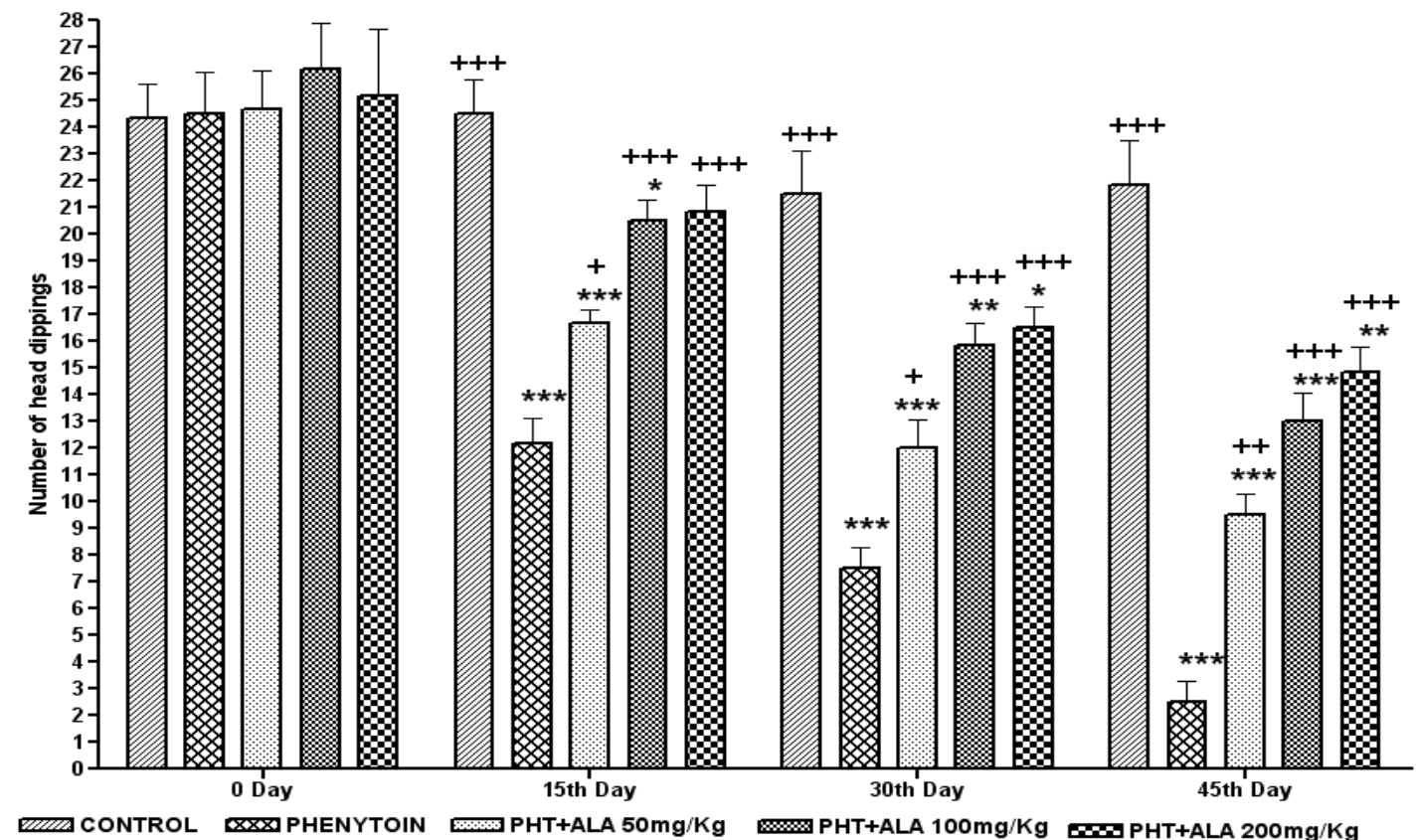

Values are expressed as mean \pm SEM of 6 animals.

${ }^{* * *}(p<0.001),{ }^{* *}(p<0.01),{ }^{*}(p<0.05)$ Vs Control group

$+++(p<0.001),++(p<0.01),+(p<0.05)$ Vs Phenytoin group

Figure 2: Effect of ALA on phenytoin impaired exploratory behaviour. 


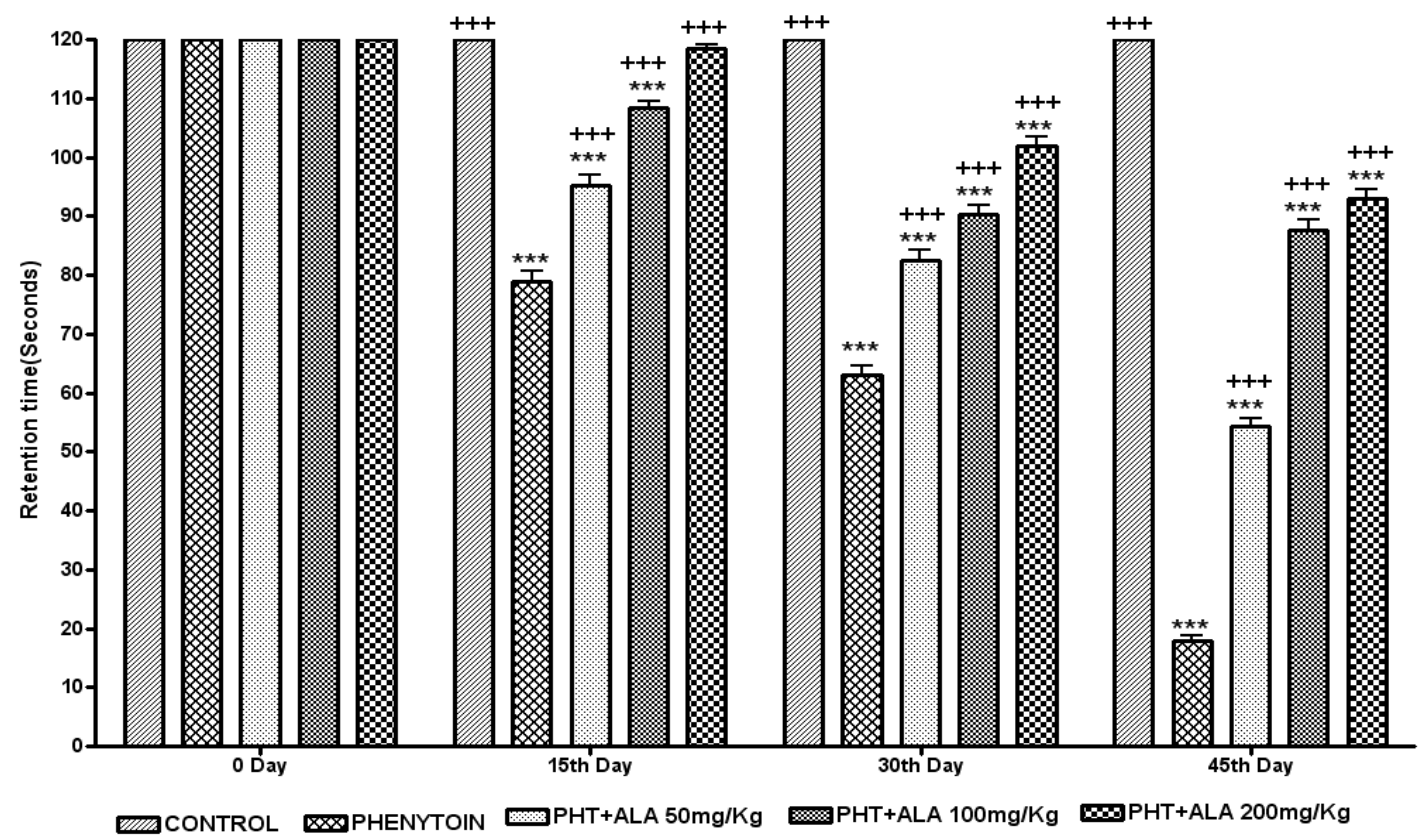

Values are expressed as mean \pm SEM of 6 animals.

${ }^{* * *}(p<0.001),{ }^{* *}(p<0.01),{ }^{*}(p<0.05)$ Vs Control group

$+++(p<0.001),++(p<0.01),+(p<0.05) \bigvee s$ Phenytoin group

Figure 3: Effect of ALA on phenytoin induced motor in-coordination.

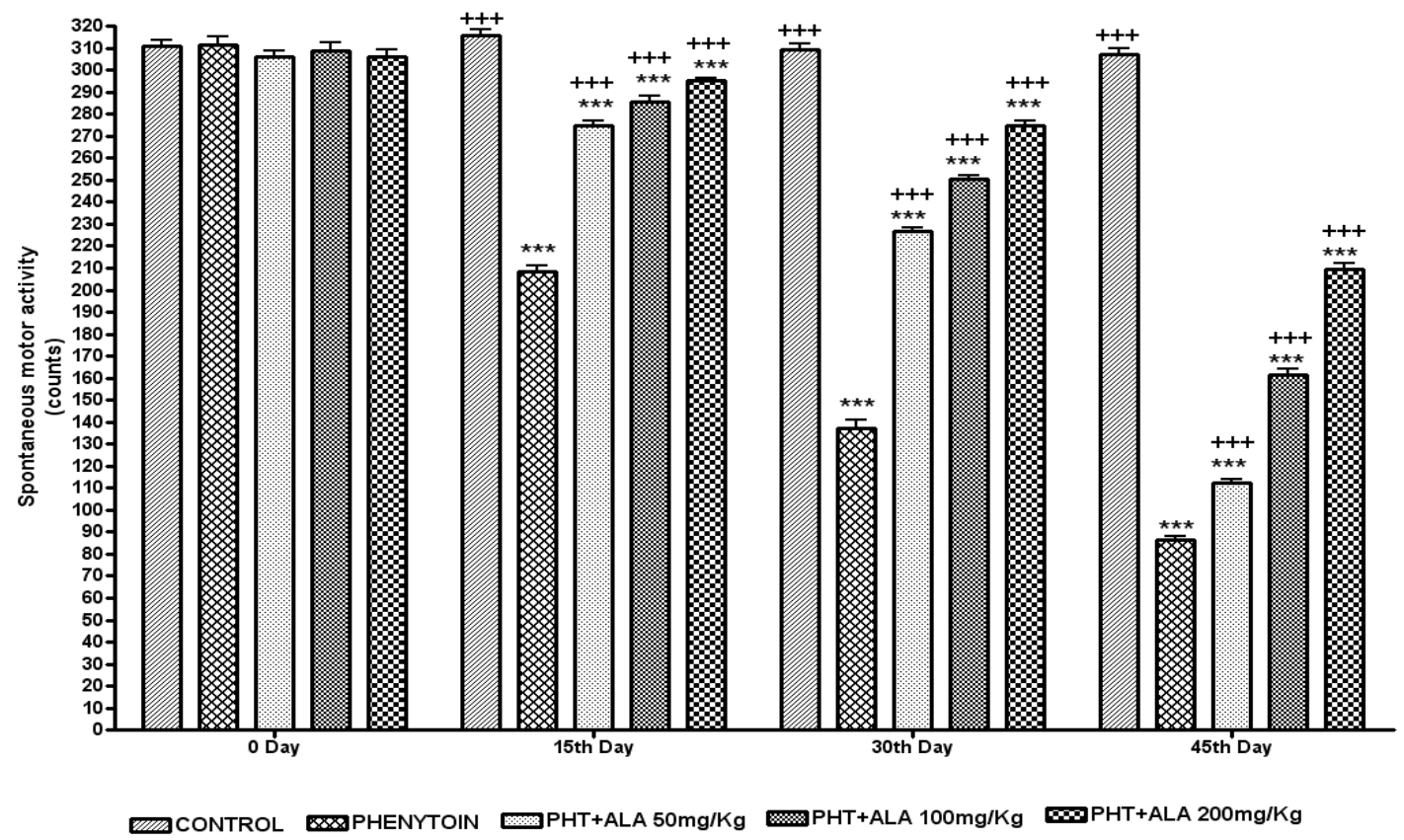

Values are expressed as mean \pm SEM of 6 animals.

${ }^{* * *}(p<0.001),{ }^{* *}(p<0.01),{ }^{*}(p<0.05)$ Vs Control group

$+++(p<0.001),++(p<0.01),+(p<0.05) \bigvee s$ Phenytoin group

Figure 4: Effect of ALA on phenytoin impaired locomotor activity. 


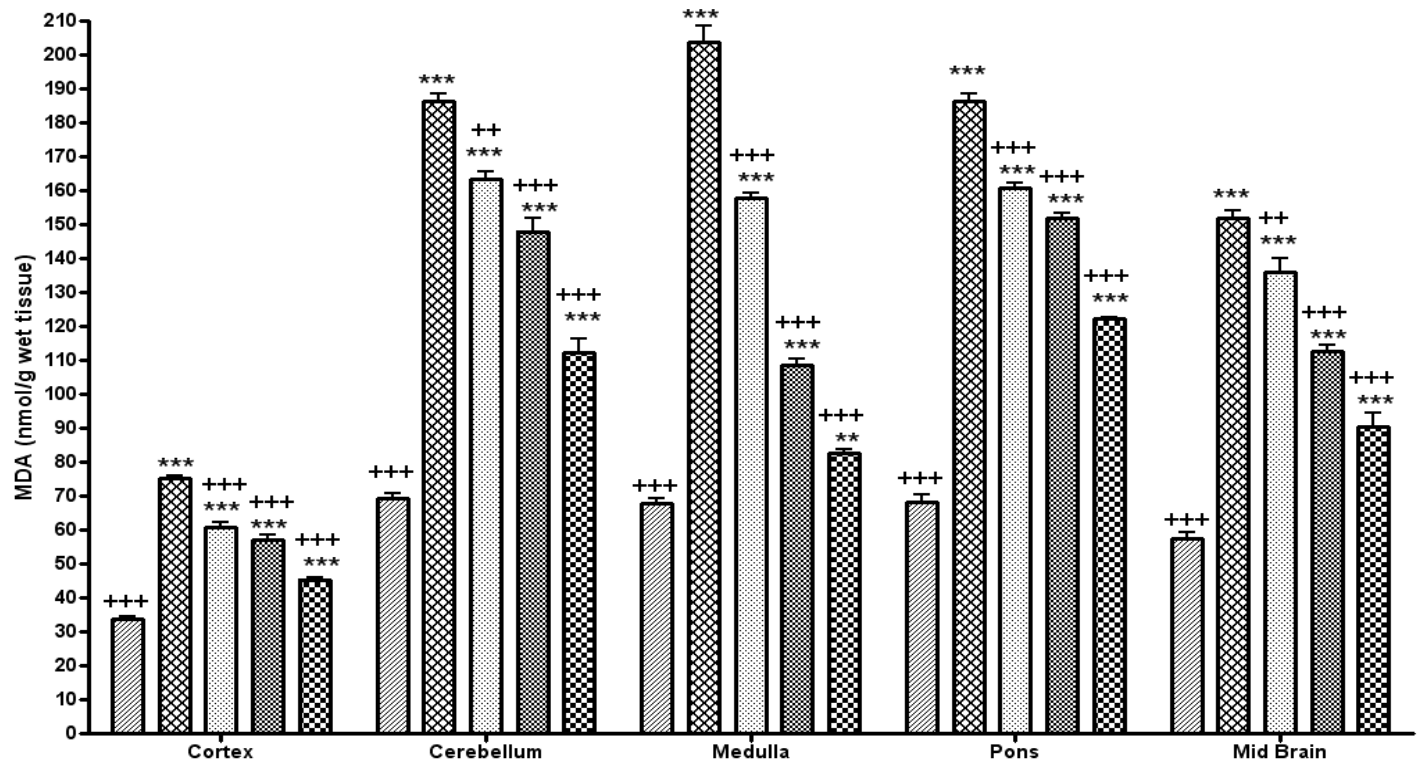

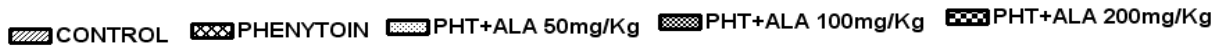

Values are expressed as mean \pm SEM of 6 animals.

${ }^{* * *}(p<0.001),{ }^{* *}(p<0.01),{ }^{*}(p<0.05)$ Vs Control group

$+++(p<0.001),++(p<0.01),+(p<0.05)$ Vs Phenytoin group

Figure 5: Effect of ALA on phenytoin induced alterations in regional brain lipid peroxidation.

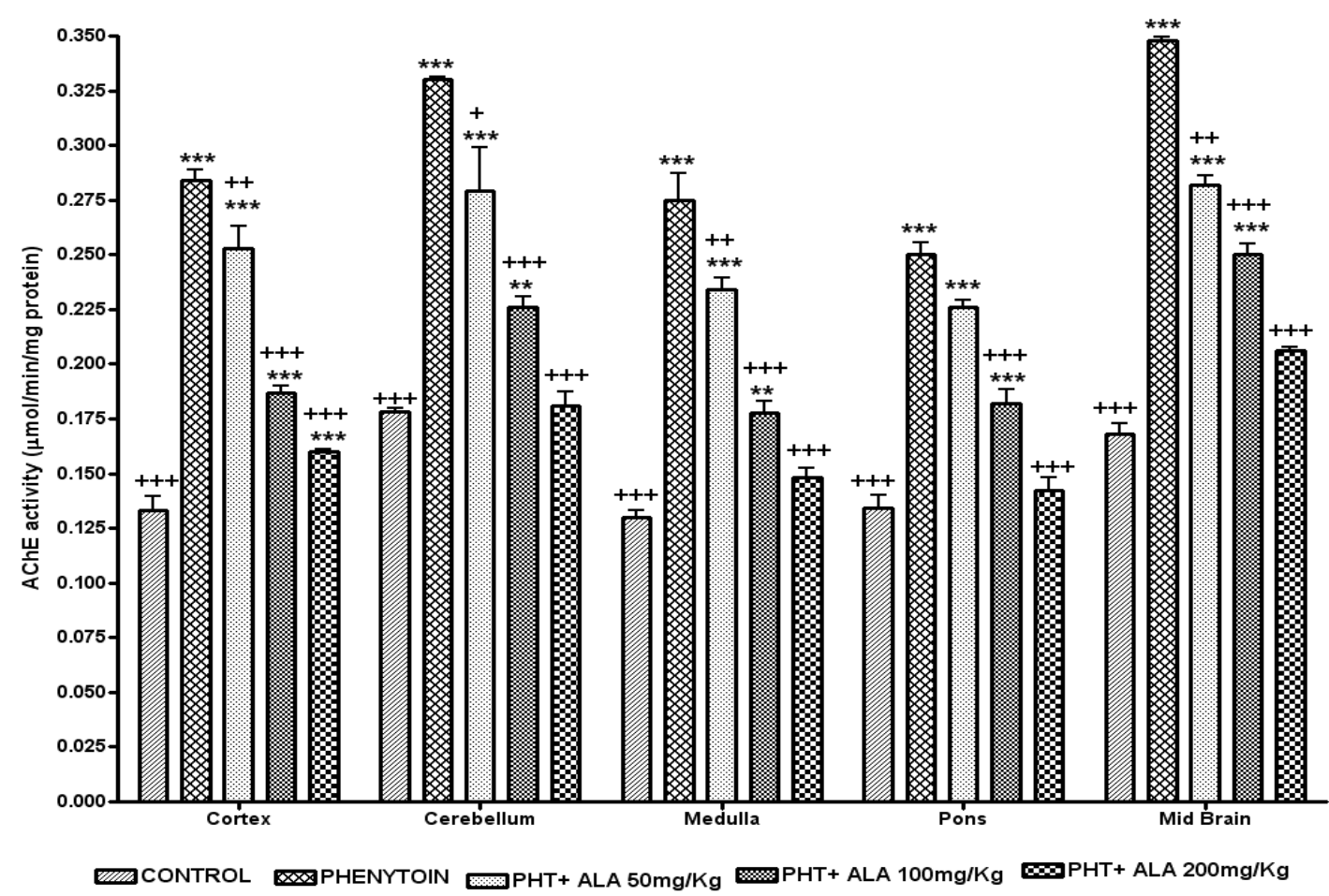

Values are expressed as mean \pm SEM of 6 animals.

${ }^{* * *}(p<0.001),{ }^{* *}(p<0.01),{ }^{*}(p<0.05)$ Vs Control group

$+++(p<0.001),++(p<0.01),+(p<0.05)$ Vs Phenytoin group

Figure 6: Effect of ALA on phenytoin induced alterations in regional brain acetyl cholinesterase activity. 

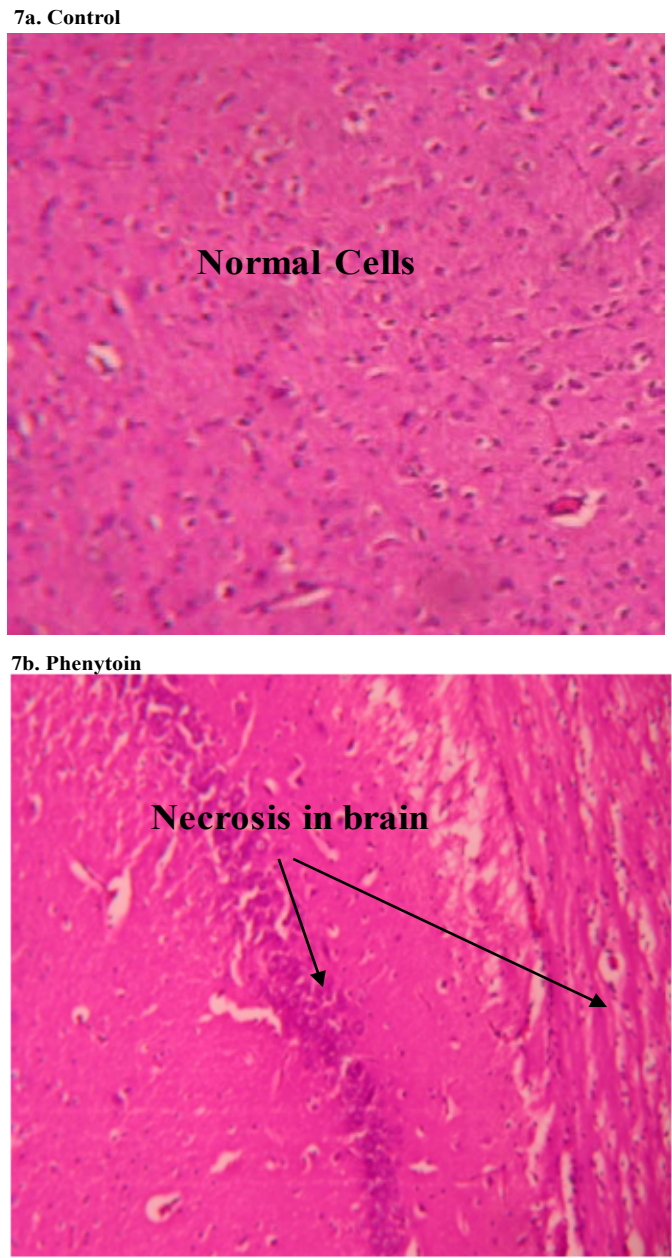

Figure 7: Effect of phenytoin on regional brain histopathology

histopathological changes in rat brain. Phenytoin in combination with $50 \mathrm{mg} / \mathrm{Kg}$ ALA showed gliosis and congestion in brain (Figure 8a), 100 $\mathrm{mg} / \mathrm{Kg}$ and $200 \mathrm{mg} / \mathrm{Kg}$ ALA showed normal brain parenchyma (Figure $8 \mathrm{~b}$ and $8 \mathrm{c})$.

\section{Influence of ALA on pharmacodynamic effect of phenytoin}

Phenytoin as well as phenytoin supplemented with ALA (50, 100 and $200 \mathrm{mg} / \mathrm{kg}$ ) offered same degree of protection (100\%) against MES induced convulsions in rats.

\section{Effect of ALA on serum phenytoin levels}

There was no significant difference in the serum concentration of phenytoin treated group as compared to the groups coadministered ALA 50, 100 and $200 \mathrm{mg} / \mathrm{kg}$ along with phenytoin. The serum phenytoin levels were $15.740 \pm 1.8,14.480 \pm 1.6,15.340$ \pm 2.2 and $15.650 \pm 1.2 \mu \mathrm{g} / \mathrm{ml}$ in the groups treated with phenytoin and ALA 50, 100 and $200 \mathrm{mg} / \mathrm{kg}$ along with phenytoin respectively. All these values were within the normal therapeutic range (10-20 $\mu \mathrm{g} / \mathrm{ml}$ ) of phenytoin.

\section{Discussion}

Phenytoin adversely affected motor-coordination, cognition, exploratory behavior and spontaneous motor activity. The drug also significantly raised the regional brain lipid peroxidation and ACh E activity along with severe degeneration in the brain regions which was revealed from the histopathological investigations.

Cognitive impairment is observed in epileptic patients and the degree of impairment depends on the number of factors in epileptic patients including the underlying pathology. Therefore, it is rational to assess the extent of AEDs induced memory and cognitive function in experimental animals without any additional complexities of the disease. Phenytoin significantly impairs the process of learning and memory consolidation [28]. Phenytoin $(20 \mathrm{mg} / \mathrm{Kg})$ was reported to substantially prolong the transfer latency of rats in elevated plus maze test, indicating the risk of this drug in impairing cognition even in healthy individuals also. Our results are online with the above finding in which, learning and memory was impaired by phenytoin in rats. Phenytoin was reported to affect the exploratory behavior [29], induce sedation and decrease the wakeful state of the rats. Phenytoin significantly decreased the exploratory behavior as there was a decrease in the number of head dippings in the holes of the hole board. Phenytoin impaired the Rota rod performance of rats indicating muscle weakness and motor in co-ordination induced by the drug. Phenytoin significantly reduced the spontaneous motor activity, indicating the CNS depressant effect of the drug.

The cerebral cortex is involved in many higher level tasks such as language, memory and consciousness. Cerebellar cortex play a critical role in long term memory storage [30] and consolidation of memory [31]. In the present study, phenytoin increased the lipid peroxidation in cerebral cortex, cerebellum, mid brain, pons and medulla oblongata. Increased lipid peroxidation in different brain regions causes peroxidative injury to the neuronal membranes and macromolecules, alter neurotransmitters, disturb neuronal functions and motor function [32]. Neuronal damage induced by phenytoin in brain regions was considered to be responsible for memory impairment, motor in co-ordination, sedation, ataxia and loss of exploratory drive.

Cholinergic activity in the CNS is essential for learning, memory, cortical organization of movement and cerebral blood flow [33] and is regulated by acetylcholine Esterase (ACh E), which hydrolyses the neurotransmitter acetylcholine ( $\mathrm{ACh}$ ) in the synaptic cleft of cholinergic synapse and neuromuscular junctions [34]. The effects of cholinergic antagonists and lesions of cholinergic nuclei are related to cognitive deficits similar to those observed in aging and dementia $[35,36]$. The antimuscarinic drug scopolamine is the most widely used to induce amnesia in experimental subjects [37]. ACh E inhibitors enhance the availability of $\mathrm{ACh}$ in the synaptic cleft and reverse the scopolamine induced memory deficit. Many studies have shown that there is a relation between the decrease in cognitive functions and markers of the cholinergic system in senile dementia [38]. Cognitive functions dependent on central cholinergic neurotransmission. Although other neurotransmitters were known to be involved in learning and memory performance, acetylcholine plays a vital role in in storage and retrieval of memory. Decline in the cholinergic system underlies the cognitive deficits of dementia [39] and ACh E levels are reported to be high in AD. Melo, et al., (2003) studied the involvement of oxidative stress in the enhancement of ACh E activity and reported that amyloid betapeptide enhanced ACh E activity mediated via oxidative stress [40].

Epileptic patients receiving phenytoin showed poor performance on memory tasks than untreated controls [41]. Investigations on the effect of phenytoin on learning, memory and psychomotor functions revealed that both acute and chronic administration of phenytoin considerably impaired learning and memory. It was reported that phenytoin decreased brain ACh levels [42]. Phenytoin's impairing 


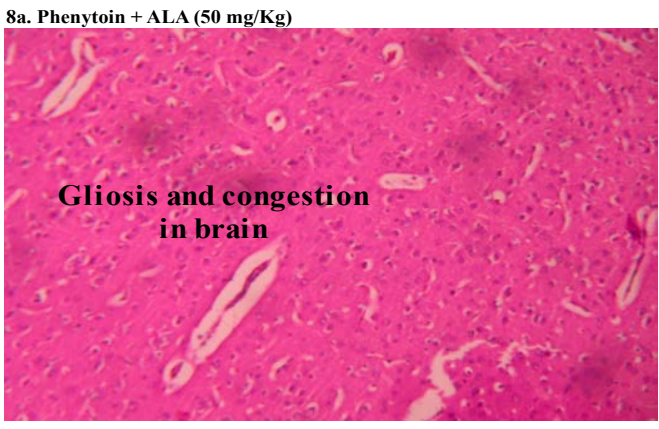

8b. Phenytoin + ALA (100 mg/Kg)

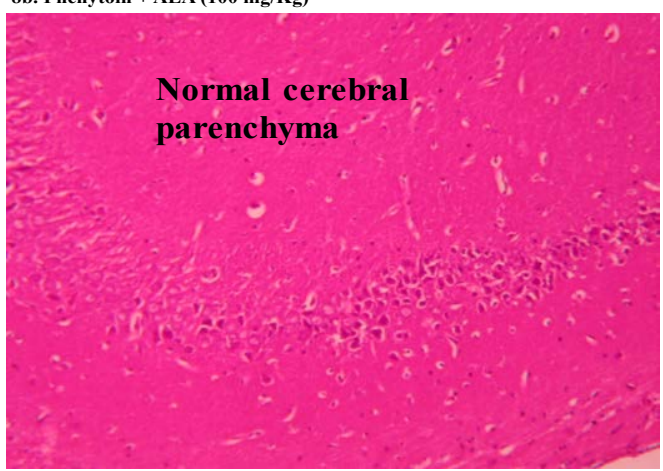

8c. Phenytoin + ALA (200 mg/Kg)

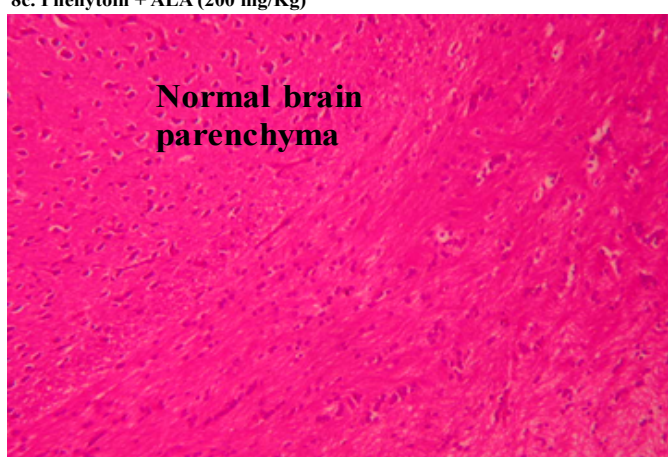

Figure 8: Effect of ALA on phenytoin induced alterations in brain histopathology.

effects on learning and memory are attributed to enhanced ACh E activity in brain. Since central cholinergic system plays an important role in learning and memory and as phenytoin reduced ACh concentration in brain regions, the drug was reported to induce serious memory impairment [42]. In the present study ACh E activity in different brain regions was measured and our results were online with the previous reports which also revealed that phenytoin at therapeutic doses increased $\mathrm{ACh} \mathrm{E}$ activity in the brain regions of the rats. The rats showed poor performance in the elevated plus maze test indicating memory impairment. It was believed that phenytoin via oxidative stress enhanced the ACh E activity and thereby depleted the levels of $\mathrm{ACh}$ in brain regions resulting in subsequent memory impairment.

Histopathological analysis of brain sections of phenytoin treated rats showed damaged cells and congestion in periventricular region and cortex, which substantiates phenytoin induced apoptosis in cortex and periventricular region. Phenytoin via oxidative stress induced the damage in rat brain, which in turn resulted in adverse behavioral abnormalities.

Since phenytoin induced oxidative stress was considered to be responsible for deterioration of behavioral parameters, the present investigation is aimed at evaluating the antioxidant potential of ALA to reverse phenytoin induced oxidative damage and behavioral abnormalities.

\section{Effect of Alpha Lipoic Acid on phenytoin induced behavioural abnormalities}

Oxidative stress was found to play a crucial role in age related neurodegenerative disorders. ALA supplementation was observed to significantly decrease haloperidol induced tardive dyskinesia and catalepsy. The protective effect of ALA against tardive dyskinesia was attributed to its ability to reduce haloperidol induced lipid peroxidation, by scavenging ROS and reactive nitrogen species. In the present study also it was found that ALA significantly increased the muscular coordination and muscle strength which was considerably reduced by phenytoin. This improvement in muscle grip was believed to be due to the antioxidant property of ALA and its protective action against oxidative stress in brain regions responsible for muscular coordination. ALA is proved to increase insulin sensitivity and activity which in turn plays a role in serotonergic activity by increasing the influx of tryptophan into the brain. This increased influx of tryptophan results in an increase in serotonin synthesis. In accordance with the serotonin theory of depression, it is possible to treat depression by increasing insulin activity. Therefore, ALA supplementation is thought to possess anti depressant activity. In the present study also it was found that ALA significantly increased the locomotor activity which was considerably reduced by phenytoin [43].

ALA derives its antioxidant capability from its ability to act as a scavenger of ROS, chelate metals and recycle endogenous antioxidants [44]. ALA scavenges singlet oxygen, $\mathrm{H}_{2} \mathrm{O}_{2}, \mathrm{OH}^{-}, \mathrm{NO}$ and ONOO. ALA and its reduced form DHLA, further scavenge oxygen and peroxyl radicals [45]. ALA also chelates several divalent cations, e.g. $\mathrm{Mn}^{2+}, \mathrm{Cu}^{2+}, \mathrm{Zn}^{2+}, \mathrm{Cd}^{2+}$ and $\mathrm{Pb}^{2+}$. Therefore, ALA inhibits ascorbate induced production of $\mathrm{H}_{2} \mathrm{O}_{2}$ by $\mathrm{Cu}^{2+}$ [46]. ALA recycles endogenous antioxidants, such as GSH [46] and Vit C [47], which in turn regenerates Vit E. GSH, Vit $\mathrm{C}$ and Vit $\mathrm{E}$ protect the brain from oxidative stress [47].

ROS species are thought to be involved in acute and chronic pathological conditions in the brain and neuronal tissue. The metabolic antioxidant ALA is a low molecular weight substance which crosses the blood brain barrier. ALA is taken up and reduced in cells and tissues to dihydro lipoic acid (DHLA), which is also exported to the extracellular medium; hence, protection is offered to both intracellular and extracellular environments. Both ALA and DHLA were considered to be potent antioxidants to regenerate other antioxidants like Vit $\mathrm{C}$ and Vit E and GSH through redox cycling. Thus, it would seem an ideal substance in the treatment of oxidative brain and neuronal disorders involving free radical processes. ALA reveals protection against cerebral ischemic reperfusion, excitotoxic amino acid brain injury, mitochondrial dysfunction, diabetes and diabetic neuropathy, inborn errors of metabolism and other causes of acute or chronic damage to brain or neuronal tissue. Antioxidant properties of ALA achieve its possible therapeutic roles in a variety of brain and neuronal tissue pathologies [48].

Acute dose of lindane causes significant reduction in butyrylcholinesterase (BChE) activity both in olfactory lobe and cerebrum of mice along with reduction in catalase, total protein and elevation in cholesterol contents. Pre-treatment by a combination of antioxidants such as Vit E, Vit C, ALA and stilbene resveratrol (125 mg/ 
Kg, ip) significantly augmented the altered level of butyrylcholinesterase and protected the other parameters in both the brain regions. The study suggested the neuroprotective efficacy of combination of antioxidants against lindane induced neurotoxicity [49].

ALA modulated AchE activity, increased choline acetyl transferase activity, improved cholinergic transmission and thus reversed the memory impairment induced by many factors such as aging, epilepsy etc. [50]. Antioxidant property of ALA was believed to combat oxidative stress induced memory impairments by improving the cholinergic transmission. The present study showed that ALA along with its antioxidant property, brought down the phenytoin elevated AchE activity, thus preserved the cholinergic transmission and improved memory.

In the present study, ALA decreased phenytoin induced lipid peroxidation and thus reversed the behavioural abnormalities induced by long term phenytoin administration. ALA $(50 \mathrm{mg} / \mathrm{Kg})$ supplementation showed Gliosis and congestion in brain, whereas ALA (100 and $200 \mathrm{mg} / \mathrm{Kg}$ ) treated group showed normal brain parenchyma. Thus ALA produced a dose dependent protective effect on phenytoin induced behavioural abnormalities and neurotoxicity.

Pharmacodynamic study was carried out to evaluate whether ALA supplementation hinders the therapeutic efficacy of phenytoin. In the present study, administration of phenytoin $(20 \mathrm{mg} / \mathrm{Kg}$ for 45 days) produced $100 \%$ protection against MES induced seizures. Coadministration of ALA with phenytoin also offered the same degree of protection against MES induced convulsions. This finding suggests that antioxidant supplementation with phenytoin did not reduce the therapeutic effect of phenytoin, revealing that there was no pharmacodynamic interaction between phenytoin and the selected antioxidants.

The serum levels of phenytoin were estimated at the end of the study period well after the steady state $(3 \mathrm{~h}$ after administration of phenytoin) of the drug was achieved. The serum levels relate to the therapeutic or toxic effects of phenytoin. It was evident from the findings of the present study that the serum levels of phenytoin were not different in the groups supplemented with ALA as compared to phenytoin alone treated group. This suggests that ALA did not alter the serum phenytoin concentration. This finding revealed that ALA did not alleviate the behavioral abnormalities by reducing serum phenytoin levels.

ALA offered protection against phenytoin induced behavioral abnormalities. The neuroprotective potential of ALA is due to its antioxidant property. Though the behavioral abnormalities were reversed, the values did not reach normal even with higher dose of ALA, indicating the involvement of other mechanisms in addition to oxidative stress in phenytoin induced behavioral abnormalities.

\section{Acknowledgment}

We thank V. Madhavan, Principal, M. S. Ramaiah College for Pharmacy and Gokula Education Foundation for their encouragement.

\section{References}

1. Delorenzo RJ, Sun DA, Deshpande LS (2005) Cellular mechanisms underlying acquired epilepsy: the calcium hypothesis of the induction and maintenance of epilepsy. Pharmacol Ther 105: 229-266.

2. McNamara JO (2006) The Pharmacological Basis of Therapeutics. Goodman and Gilman's Mc Graw-Hill.

3. Raya A, Gallego J, Bosch-Morell F (1995) Phenytoin induced glutathione depletion in rat peripheral nerve. Free Radical Biology Med 19; 665-667.
4. Luef G, Chemelli A, Birbamer G, Aichner F, Bauer G (1994) Phenytoin overdosage and cerebellar atrophy in epileptic patients: clinical and MRI findings. European Neurology 34: 79-81.

5. Pandhi P, Balakrishnan S (1999) Cognitive dysfunction induced by phenytoin and valproate in rats: effect of nitric oxide. Indian Journal Physiology and Pharmacology 43: 378-382.

6. Reynolds EH (1975) Chronic antiepileptic toxicity: a review. Epilepsia 16: 319 352.

7. Golbidi S, Badran M, Laher I (2011) Diabetes and alpha lipoic Acid. Front Pharmacol 2: 69 .

8. Shay KP, Moreau RF, Smith EJ, Smith AR, Hagen TM (2009) Alpha-lipoic acid as a dietary supplement: molecular mechanisms and therapeutic potential. Biochim Biophys Acta 1790: 1149-1160.

9. Bock E, Schneeweiss J (1959) Ein Beitrag zur Therapie der neuropathia diabetic. Munchner Med Wochenschrift 43: 1911-1912.

10. Wray DW, Nishyyama SK, Harris RA, Zhao J, McDaniel J, et al. (2012) Acute reversal of endothelial dysfunction in the elderly following antioxidant consumption. Hypertension 59: 818-824.

11. McNeilly AM, Davison GW, Murphy MH, Nadeem N, Trinick T, et al. (2011) Effect of a-lipoic acid and exercise training on cardiovascular disease risk in obesity with impaired glucose tolerance. Lipids Health Dis 10: 217.

12. Zhang WJ, Bird KE, McMillen TS, LeBoeuf RC, Hagen TM, et al. (2008) Dietary alphalipoic acid supplementation inhibits atherosclerotic lesion development in apolipoprotein E-deficient and apolipoprotein E/low-density lipoprotein receptor-deficient mice. Circulation 117: 421-428.

13. Ying Z, Kherada N, Farrar B, Kampfrath T, Chung Y, et al. (2010) Lipoic acid effects on established atherosclerosis. Life Sci 86: 95-102.

14. Ziegler D, Reljanovic M, Mehnert H, Gries FA (1999) a Lipoic acid in the treatment of diabetic polyneuropathy in Germany: current evidence from clinical trials. Exp Clin Endocrinol Diabetes 107: 421-430.

15. Packer L, Kraemer K, Rimbach G (2001) Molecular aspects of lipoic acid in the prevention of diabetes complications. Nutrition 17: 888-895.

16. Vasdev S, Ford CA, Parai S, Longerich L, Gadag V (2000) Dietary alpha-lipoic acid supplementation lowers blood pressure in spontaneously hypertensive rats. J Hypertens 18: 567-573.

17. Moreira PI, Harris PLR, Zhu X, Santos MS, Oliveira CR, et al. (2007) Lipoic acidi and n-acetyl cysteine decrease mitochondrial-related oxidative stress in Alzheimer disease patient fibroblasts. J Alzheimers Dis 12: 195-206.

18. Lott IT, Doran E, Nguyen VQ, Tournay A, Head E, et al. (2011) Down syndrome and dementia: a randomized, controlled trial of antioxidant supplementation. Am J Med Genet 155A: 1939-1948.

19. Al Abdan M (2012) Alfa-lipoic acid controls tumor growth and modulates hepatic redox state in Ehrlich-ascites-carcinoma-bearing mice. Scientific World Journal 2012: 509838

20. Sharma AC, Kulkarni SK (1992) Evaluation of learning and memory mechanisms employing elevated plus maze in rats and mice. Progress in Neuro-Psychopharmacology and Biological Psychiatry 16: 117-125.

21. Kulkarni SK (1999) Hand book of experimental pharmacology. Vallabh Prakashan.

22. Takeda H, Tsuji M, Matsumiya T (1998) Changes in head-dipping behaviou in the hole-board test reflect the anxiogenic and/ or anxiolytic state in mice. European Journal of Pharmacology 350: 21-29.

23. Glownski J, Iversen LL (1966) Regional studies of catecholamines in the rat brain. The disposition of $[3 \mathrm{H}]$ norepinephrine, $[3 \mathrm{H}]$ dopamine and $[3 \mathrm{H}]$ dopa in various regions of the brain. Journal of Neurochemistry 13: 655-669.

24. Ohkawa H, Ohishi N, Yagi K (1979) Assay for lipid peroxides in animal tissue by thiobarbituric acid reaction. Analytical Biochemistry 95: 351-358.

25. Ellman GL, Courtney KD, Andres V, Feather-Stone RM (1961) A new and rapid colorimetric determination of acetylcholinesterase activity. Biochemical Pharmacology 7: 88-95.

26. Li Y, Powers C, Jiang N, Chopp M (1998) Intact, injured, necrotic and apoptotic cells after focal cerebral ischemia in the rat. Journal of Neurological Sciences 156: 119-132. 
Citation: Saraswathy GR, Maheswari E, Santhrani T (2015) Protective Effect of Alpha Lipoic Acid against Phenytoin Induced Behavioral Abnormalities in Rats. J Mol Biomark Diagn 5: 241. doi:10.4172/2155-9929.1000241

27. Chen LC, Chou MH, Lin MF, Yang LL (2001) Effects of Paeoniae radix, a traditional Chinese medicine, on the pharmacokinetics of phenytoin. Journal of Clinical Pharmacy and Therapeutics 26: 271-178.

28. Santhrani Thaakur, Pushpa KB (2008) Influence of spirulina on phenytoin induced selected behavioural abnormalities and regional brain lipid peroxidation in rats. International Journal of Neurodegeneration and Neuroprotection 4: 263-272.

29. Bala Krishnan S, Bhargava VK, Padhi P (1998) Effect of nimodipine on the psychomotor dysfunction induced by phenytoin rats. Indian Journal of Pharmacology 30: 299-305.

30. Christian KM, Thompson RF (2005) Long-term storage of an associative memory trace in the cerebellum. Behavioral Neuroscience 119: 526-537.

31. Fliessbach K, Trautner P, Quesada CM, Elger CE, Weber B (2007) Cerebellar contributions to episodic memory encoding as revealed by fMRI. Neurolmage 35: $1330-1337$

32. Markesbery WR (1997) Oxidative stress hypothesis in Alzheimer's disease. Free Radical Biology and Medicine 23: 134-147.

33. Mesulam MM, Guillozet A, Shaw P, Levey A, Duysen EG, et al. (2002) Acetylcholinesterase knockouts establish central cholinergic pathways and can use butyrylcholinesterase to hydrolyze acetylcholine. Neuroscience 110: 627-639.

34. Schmatz R, Mazzanti CM, Spanevello R, Stefanello N, Gutierres J, et al. (2009) Resveratrol prevents memory deficits and the increase in acetylcholinesterase activity in streptozotocin-induced diabetic rats. European Journal of Pharmacology 610: 42-48

35. Dawson GR, Heyes CM, Iversen SD (1992) Pharmacological mechanisms and animal models of cognition. Behavioural Pharmacology 3: 285-297.

36. Molchan SE, Martinez RA, Hill JL, Weingartner HJ, Thompson K, et al. (1992) Increased cognitive sensitivity to scopolamine with age and a perspective on the scopolamine model. Brain Research Reviews 17: 215-226.

37. Drachman DA, Leavitt $\mathrm{J}$ (1974) Human memory and the cholinergic system: a relationship to aging?. Archives of Neurology 30: 113-121.

38. Perry EK, Tomlinson BE, Blessed G, Bergmann K, Gibson PH, et al. (1978) Correlation of cholinergic abnormalities with senile plaques and mental test scores in senile dementia. British Medical Journal 2: 1457-1459.
39. Blockland A (1996) Acetylcholine: a neurotransmitter for learning and memory? Brain Research Reviews 21: 285-300.

40. Melo JB, Agostinho P, Oliveira CR (2003) Involvement of oxidative stress in the enhancement of acetylcholinesterase activity induced by amyloid beta-peptide. Neuroscience Research 45: 117-127.

41. Andrewes DG, Bullen JG, Tomlinson L, Elwes DC, Reynolds EH (1986) A comparative study of the cognitive effects of phenytoin and carbamazepine in new referrals with epilepsy. Epilepsia 27: 128-134.

42. Domino EF, Olds ME (1972) Effects of D-amphetamine, scopolamine, chlordiazepoxide and diphenylhydantoins on self-stimulation and brain acetylcholine. Psychopharmacologia 23: 1-16.

43. Thaakur S, Himabindu G (2009) Effect of alpha lipoic acid on the tardive dyskinesia and oxidative stress induced by haloperidol in rats. J Neural Transm 116: 807-814.

44. Lynch MA (2001) Lipoic acid confers protection against oxidative injury in nonneuronal and neuronal tissue. Nutr Neurosci 4: 419-438.

45. Kagan VE, Shvedova A, Serbinova E, Khan S, Swanson C, et al. (1992) Dihydrolipoic acid - a universal antioxidant both in the membrane and in the aqueous phase. Reduction of peroxyl, ascorbyl and chromanoxyl radicals. Biochem Pharmacol 44: 1637-1649.

46. Ou P, Tritschler HJ, Wolff SP (1995) Thioctic (lipoic) acid: a therapeutic metalchelating antioxidant?. Biochem Pharmacol 50: 123-126.

47. Drake J, Kanski J, Varadarajan S, Tsoras M, Butterfield DA (2002) Elevation of brain glutathione by gamma-glutamylcysteine ethyl ester protects against peroxynitrite-induced oxidative stress. J Neurosci Res 68: 776-784.

48. Packer L, Tritschler HJ, Wessel K (1997) Neuroprotection by the metabolic antioxidant alpha-lipoic acid. Free Radic Biol Med 22: 359-378.

49. Aliev G, Liu J, Shenk JC, Fischbach K, Pacheco GJ, et al. (2009) Neurona mitochondrial amelioration by feeding acetyl-L-carnitine and lipoic acid to aged rats. J Cell Mol Med 13: 320-333.

50. Arivazhagan P, Ayusawa D, Panneerselvam C (2006) Protective efficacy of alpha-lipoic acid on acetylcholinesterase activity in aged rat brain regions. Rejuvenation Res 9: 198-201. 